\title{
ON THE OPTIMALITY OF A SWITCH-OVER POLICY FOR CONTROLLING THE \\ QUEUE STZE IN AN M/G/1 QUEUE WTTH VARIABLE SERVICE RATE
}

\author{
Henk Tijms \\ Mathematisch Centrum \\ Ansterdam
}

\section{ABSTRACT}

This paper considers an $M / G / 1$ queve in which a finite number of service types are available for controlling the queue size. There is a linear holding cost, a service cost rate, and a fixed reward for each customer served. The purnose of this paper is to show that under the assumption of stochasticallv ordered service times there is an average cost optimal stationary nolicy having the property that the service type used is a non-decreasing function of the queue size.

\section{INTRODUCTION}

Consider a single-wserver station where customers arrive in accordance with a Poisson process with rate $\lambda$. For each new service to be started the server must choose one of a finite number of different service types $k=1, \ldots, M$. For service type $k$ the service time is a positive random variable $s_{k}$ with probability distribution function $F_{k}(t)$. It is assumed that $s_{k}$ is stochastically smaller than $s_{j}$ for all $k$ and $j$ with $k>j$, that is, $F_{k}(t) \geq F_{j}(t)$ for $t \geq 0$ when $k>j$, so tvpe $k$ is "faster" than type $j$ for $k>j$. Further we assume that $\lambda \mathrm{ES}_{\mathrm{M}}<1$ and $\mathrm{ES}_{\mathrm{k}}^{2}<\infty$ for all $\mathrm{k}$. The following costs are considered. There is a holding cost of $h>0$ per customer per unit time, a service cost rate $r_{k} \geq 0$ when the server is busy and uses service type $k$, a service cost at rate $r_{0} \geq 0$ when the server is idle, and a fixed reward of $R_{k} \geq 0$ for each customer served by using service type $k$.

Define the state of the system as the number of customers present. The system is only observed at the epochs where a new service must be started and the enochs where the server becomes idle. When the system is observed in state $i \geq 1$, then one of the actions $\mathrm{k}=1, \ldots, \mathrm{M}$ must be chosen where the choice of action $\mathrm{k}$ means that service type $k$ is used for the new service to be started. For notational purposes, we say that action 0 is chosen when state 0 is observed. Let $c(i, k)$ be the expected cost incurred until the next review when in state $i$ action $k$ is chosen. Then, $C(i, k)=$ $=h i E S_{k}+h \lambda \mathrm{ES}_{\mathrm{k}}^{2} / 2+r_{k} \mathrm{ES}_{\mathrm{k}}-\mathrm{R}_{\mathrm{k}}$ for $\mathrm{all} \mathrm{i} \geq 1$ and $1 \leq \mathrm{k} \leq \mathrm{M}$, and $\mathrm{C}(0,0)=\mathrm{r}_{0} / \lambda$.

Since we will consider the average cost criterion, it is no restriction to assume that immediate costs $C(i, k)$ are incurred when action $k$ is taken in state $i$. A policy $\pi$ is any rule for choosing actions, where a policy $f$ is said to be stationary

*) This paper appeared as Mathematical Centre Renort BN 25/75. 
if it chooses a single action $f(i)$ whenever the system is in state $i$. A stationary policy $f$ is called a switch-over policy when $f(i)$ is non-decreasing in $i \geq 1$.

Let $V(\pi, i, t)$ be the total expected cost incurred in $\lceil 0, t)$ when nolicy $\pi$ is used and the initial state $i$, and, for any $i$ and $\pi$, let

$$
\bar{V}(\pi, i)=\limsup _{t \rightarrow \infty} t^{-1} V(\pi, i, t) \text { and } V_{\alpha}(\pi, i)=\int_{0}^{\infty} e^{-\alpha t} d V(\pi, i, t)
$$

for $\alpha>0$, so, for initial state $i$ and policy $\pi, \bar{v}(\pi, i)$ is the long-run average cost and $V_{\alpha}(\pi, i)$ is the expected total discounted cost when the discount factor is $\alpha$. A policy $\pi^{*}$ is called average cost optimal when $\vec{V}\left(\pi^{*}, i\right) \leq \bar{V}(\pi, i)$ for all $i$ and $\pi$, and a policy $\pi^{*}$ is called $\alpha$-optimal when $V_{\alpha}\left(\pi^{*}, \mathbf{i}\right) \leq V_{\alpha}(\pi, i)$ for all $i$ and $\pi$. Let $v_{\alpha}(i)=$ $=\inf _{\pi} V_{\alpha}(\pi, i), i \geq 0$.

The existence of an average cost optimal switch-over policy was shown in CRABILL [3], LIPPMAN $[8,9]$ and SOBEL $\lceil 14]$ for the case where the service times are exponential and the service rate can also be chosen at arrival epochs, cf. also BEJA \& TELLER [1]. SCHASSBERGER [13] considered the case of stochastically ordered service times and, assuming a finite waiting room and no holding cost, he proved that there is an average cost optimal switch-over policy. His proof, however, fails for the model of this paper. The purpose of this paper is to demonstrate that using recent work of LIPPMAN $[8,10]$ the average cost optimality of a switch-over policy can be readily shown. It seems reasonable to conjecture that the results of this paper also hold under the weaker assumption of decreasing mean service times. However, this case will probably require a quite different argumentation. A more complex policy will be optimal when there are fixed costs for switching from one service rate to another. This problem has been studied in CRABILL $\lceil 4\rceil$ under the assumption of exponential service times. In the above references the control variable is the queue size. For the case where the control variable is the total amount of work remaining to be processed in the system related work was done in DOSHI [5], MITCHELL $\lceil 117$, THATCHER [15] and TIJMS [16].

\section{PROOF}

We first give some preliminaries. The notation $\mathrm{X} \subset \mathrm{Y}$ means that the random variable $X$ is stochastically smaller than the random variable $Y$. We have (see [17]) LEMMA 1 . Let $\mathrm{X} \subset \mathrm{X}$. Then, for any non-decreasing function $\mathrm{f}, \mathrm{Ef}(\mathrm{X}) \leq \mathrm{Ef}(\mathrm{Y})$ provided the expectations exist.

Let $A_{k}$ be distributed as the number of arrivals during a service time $S_{k}$. Since $\mathrm{P}\left\{\mathrm{A}_{\mathrm{k}}>\mathrm{n}\right\}=\int_{0}^{\infty} \mathrm{P}\left\{\mathrm{A}_{\mathrm{k}}>\mathrm{n} \mid \mathrm{S}_{\mathbf{k}}=\mathrm{t}\right\} \mathrm{dF}_{\mathrm{k}}(\mathrm{t})$, lemma 1 implies LEMMA 2. $A_{k} \subset A_{j}$ for all $k$ and $j$ with $k>j$. 
Denote by $z_{1}(t)$ the total holding and service costs incurred in $\left.\Gamma 0, t\right)$ and denote by $Z_{2}(t)$ the total rewards received for servicing customers during $(0, t)$. Then $z(t)=z_{1}(t)-z_{2}(t)$ is the total cost incurred in $\left.\Gamma 0, t\right)$. Let $T$ be the epoch of the first return of the system to state 0 . Denote by $E_{i, \pi}$ the expectation when policy $\pi$ is used and the initial state is $i$.

LEMMA 3. Let $\mathrm{f}$ be a stationary policy such that $\mathrm{f}(\mathrm{i})=\mathrm{M}$ for all $\mathrm{i}$ sufficiently large. Then both $\mathrm{E}_{\mathrm{i}, \mathrm{f}}(\mathrm{T})$ and $\mathrm{E}_{\mathrm{i}, \mathrm{f}}\left(\mathrm{Z}_{2}(\mathrm{~T})\right.$ ) are bounded by a linear function of $\mathrm{i}$ and $\mathrm{E}_{i, f}\left(Z_{1}(\mathrm{~T})\right)$ is bounded by a quadratic function of $i$.

PROOF. Consider the basic M/G/1 queue in which the traffic intensity is less than 1 and the service time has a finite second moment. Suppose that at epoch 0 a service starts when $s \geq 1$ customers are present. From queueing theory it is we11 known that both the expectation of the first epoch at which the system becomes empty and the expected number of customers served up to that epoch are linear functions of s. Further the expected total time spent in the system by the customers up to the first epoch at which the system becomes empty is a quadratic function of $\mathrm{s}$. Since for our model $\lambda \mathrm{ES}_{\mathrm{M}}<1$ and $\mathrm{ES}_{\mathrm{M}}^{2}<\infty$, the lemma now follows easily.

LEMMA 4. Consider the basic M/G/1 queue with $\lambda \mathrm{ES}<1$ and $\mathrm{ES}^{2}<\infty$ where $\lambda$ is the arrival rate and $S$ is the service time of a customer. Denote by $N_{n}$ the number of customers present when the nth service starts. Then $\mathrm{EN}_{\mathrm{n}}^{2} / \mathrm{n}$ converges to 0 as $\mathrm{n}+\infty$.

PROOF. Denote by $L_{n}$ the number of customers present just after the nth service completion epoch. Further, let $\nu_{k+1}$ be the number of customers arriving during the service time of the $k$ th customer, let $\delta(0)=0$, and let $\delta(x)=1$ for $x>0$. Since $0 \leq N_{n} \leq L_{n-1}+1$ for $n \geq 2$, it suffices to prove that $\mathrm{EL}_{n}^{2} / \mathrm{n}$ converges to 0 as $\mathrm{n} \rightarrow \infty$. Now this follows easily by working out the obvious identity

$$
\frac{1}{n} \sum_{k=1}^{n} E L_{k+1}^{2}=\frac{1}{n} \sum_{k=1}^{n} E\left(L_{k}-\delta\left(I_{k}\right)+v_{k+1}\right)^{2} \quad \text { for } n \geq 1
$$

and using the fact that $1 i_{n \rightarrow \infty} E I_{n}=\lambda E S+\lambda^{2} E S^{2} / 2(1-\lambda E S)$ and $\lim _{n \rightarrow \infty} E \delta\left(L_{n}\right)=\lambda E S$ (e.g. COHEN [2]),

I am indebted to Professor J.W. COHEN for suggesting the proof of this Lemma.

To prove that there is an average cost optimal switch-over policy, we first consider the discounted model. For the semi-Markov decision model with unbounded costs HARRISON $[6,7]$ and IIPPMAN $[8,10]$ have given conditions under which for each $\alpha>0$ an $\alpha$-optimal stationary policy exists and the optimality equation anplies. It is straightforward to verify that for this problem both the conditions in $[6]$ and those in [10] hold. This implies that for any $\alpha>0$ and $i \geq 1$,

$$
v_{\alpha}(i)=\min _{1 \leq k \leq M}\left\{C(i, k)+\int_{0}^{\infty} e^{-\alpha t} \sum_{j=0}^{\infty} v_{\alpha}(i-1+j) e^{-\lambda t \frac{(\lambda t) j}{j !}} d F_{k}(t)\right\},
$$


where $V_{\alpha}(0)=r_{0} / \lambda+\int_{0}^{\infty} e^{-\alpha t} v_{\alpha}(1) \lambda e^{-\lambda t} d t$. Also, for any $\alpha>0$, let $f_{\alpha}$ be a stationary policy such that $f_{\alpha}(i)$ minimizes the right side of (1) for all $i$, then $f_{\alpha}$ is a-optimal. Using lemmas 1 and 2 and making a minor modification of the first part of the proof of Theorem 6 in [8], we get that there is an $\alpha^{*}>0$ and a bound $B<\infty$ such that $f_{\alpha}(i)=M$ for all $0<\alpha<\alpha^{*}$ and $i>B$. This implies

LEMMA 5. There is a stationary polioy $\mathrm{f}^{*}$ with $\mathrm{f}^{*}(\mathrm{i})=\mathrm{M}$ for all $\mathrm{i}>\mathrm{B}$ and a sequence $\left\{\alpha_{k}\right\}$ with $\alpha_{k} \rightarrow 0$ as $k \rightarrow \infty$ such that $\mathrm{f}_{\alpha_{k}}=f^{*}$ for all $\mathrm{k}$.

The next theorem can be readily obtained from a close examination of the analysis of the average cost criterion in $\lceil 8,107$. However, since this analysis is rather complicated by its generality and needs some modifications, it might be helpful to outline a simple proof that suffices for the present problem.

THEOREM 1. The policy $\mathrm{f}^{*}$ is average cost optimal, and $\bar{v}\left(\mathrm{f}^{*}, \mathrm{i}\right)=\mathrm{g}$ for all i for some constant $\mathrm{g}$. There is a function $\mathrm{h}$ with $\mathrm{h}(\mathrm{O})=0$ and

$$
|h(\mathbf{i})| \leq \alpha i^{2}+\beta \mathbf{i}+\gamma \quad \text { for } i \geq 0,
$$

for some constants $\alpha$, $\beta$ and $\gamma$, such that $h(0)=r_{0} / \lambda-g / \lambda+h(1)$ and

$$
h(i)=\min _{1 \leq k \leq M}\left\{C(i, k)-g E S_{k}+\sum_{j=0}^{\infty} h(i-1+j) g(k)\right\} \text { for } i \geq 1,
$$

where $\mathrm{P}_{\mathrm{j}}^{(\mathrm{k})}=\mathrm{P}\left\{\mathrm{A}_{\mathrm{k}}=\mathrm{j}\right\}=\int_{0}^{\infty} \mathrm{e}^{-\lambda \mathrm{t}}\left\{(\lambda \mathrm{t})^{\mathrm{j}} / \mathrm{j}\right\} \mathrm{d \textrm {dF } _ { \mathrm { k } }}(\mathrm{t})$. Moreover, $\mathrm{f}^{\star}(\mathrm{i})$ minimizes the right side of (3) for alz i.

PROOF. Let $g=E_{0, f^{\star}}(Z(T)) / E_{0, f^{\star}}(T)$. Then, by Lemma 3 and Theorem 3.16 in ROSS $\lceil 127$, we have that $t^{-1} V\left(f^{*}, i, t\right)$ has the finite limit $g$ as $t \rightarrow \infty$ for all $i$. Now, from Lemma 5 and a standard Abelian theorem (see pp.181-182 in [187) it follows that, for all $i$ and $\pi$,

$$
\begin{aligned}
\bar{V}(\pi, i) & =\underset{t \rightarrow \infty}{\lim \sup } t^{-1} V(\pi, i, t) \geq \underset{a \rightarrow 0}{\lim \sup \alpha V_{\alpha}(\pi, i) \geq} \\
& \geq \limsup _{k \rightarrow \infty} \alpha_{k} V_{\alpha_{k}}(i)=\lim _{k \rightarrow \infty} \alpha_{k} V_{\alpha}\left(f^{*}, i\right)=\lim _{t \rightarrow \infty} t^{-1} V\left(f^{\star}, i, t\right) .
\end{aligned}
$$

This proves the first part of the theorem. As a byproduct we find

$$
\lim _{k \rightarrow \infty} \alpha_{k} V_{\alpha_{k}}(i)=g \quad \text { for all } i \geq 0
$$

Following the proof of Theorem 4 in $[8]$ (cf. also p.148 in $\lceil 127$ ) and using (4), we find that, for some constant $\delta$,

$$
-E_{i, f^{\star}}\left(Z_{2}(T)\right)+\delta E_{i, f^{*}}(T) \leq v_{\alpha_{k}}(i)-v_{\alpha_{k}}(0) \leq E_{i, f^{*}}\left(Z_{1}(T)\right) \text { for all } k, i
$$


So, for each $\mathrm{i},\left\{\mathrm{v}_{\alpha_{k}}(\mathrm{i})-\mathrm{v}_{\alpha_{k}}(0)\right\}$ is a bounded sequence. Now by Cauchy's diagonalization method, there is a subsequence $\left\{\alpha_{k}^{1}\right\}$ of $\left\{\alpha_{k}\right\}$ and a function $h$ such that

$$
h(i)=\lim _{k \rightarrow \infty}\left\{V_{a_{k}^{\prime}}(i)-V_{\alpha_{k}^{\prime}}(0)\right\} \quad \text { for all } i \geq 0
$$

By (5), (6) and Lemma 3 we have that h satisfies (2). Next we observe that, by (2) and $\mathrm{ES}_{\mathrm{k}}^{2}<-\infty$,

$$
\sum_{j=0}^{\infty}|h(i-1+j)| p_{j}^{(k)}<\infty \quad \text { for all } i \geq 1 \text { and } 1 \leq k \leq M \text {. }
$$

Finally, subtracting $V_{\alpha}(0)$ from both sides of (1) with $\alpha=\alpha_{k}^{\prime}$, letting $k \rightarrow \infty$, and using (4), (6), (7) and the construction of $f^{*}$, we find the other assertions of the theorem (we note that (7) is needed for applying the bounded convergence theorem).

A repetition of the second part of the nroof of Theorem 6 in 587 shows LEMMA 6. $h(i+1)-h(i) \geq h(i)-h(i-1)$ for alz $i \geq 1$, i.e. the function $h$ is convex.

Denote by $h(i, k)$ the expression between brackets in (3). Then

LEMMA 7. EOr all $\mathrm{k}_{1}, \mathrm{k}_{2}$ with $\mathrm{k}_{1}>\mathrm{k}_{2}, \mathrm{~h}\left(\mathrm{i}, \mathrm{k}_{1}\right)-\mathrm{h}\left(\mathrm{i}, \mathrm{k}_{2}\right)$ is non-increasing in $\mathrm{i} \geq 1$. PROOF. Fix $k_{1}, k_{2}$ with $k_{1}>k_{2}$ and fix $i \geq 1$. Using (7), we have

$$
\begin{aligned}
h\left(i+1, k_{1}\right) & -h\left(i+1, k_{2}\right)-\left\{h\left(i, k_{1}\right)-h\left(i, k_{2}\right)\right\}=h E S_{k_{1}}-h E S_{k_{2}}+ \\
& \left.+\sum_{j=0}^{\infty}\{h(i+j)-h(i-1+j)\}_{j}\left(k_{1}\right)-\sum_{j=0}^{\infty}\{h(i+j)-h(i-1+j)\} p_{i} k_{2}\right)
\end{aligned}
$$

By lemma $6, h(i+j)-h(i-1+j)$ is non-decreasing in $j \geq 0$. Now, the lemma follows from the Lemmas 1 and 2 and the fact that $\mathrm{ES}_{\mathrm{k}_{1}}<\mathrm{ES}_{\mathrm{k}_{2}}$.

We are now in a position to state our main result.

THEOREM 2. For any $i \geq 1$, let $\mathrm{f}_{0}(\mathrm{i})$ be the largest value of $\mathrm{k}$ for which the right side of (3) is minimal. Then, $\mathrm{f}_{0}$ is an average cost optimal switch-over policy which uses service type $M$ for all i sufficiently large.

PROOF. It easily follows from Lemma 7 that $f_{0}(i+1) \geq f_{0}(i)$ for all $i \geq 1$, and, by Lemma 5 and Theorem $1, f_{0}(i)=M$ for all $i$ sufficiently large. Since $g$ is the minimal average cost, the switch-over policy $f_{0}$ is optimal when $\bar{v}\left(f_{0}, i\right)=g$ for all $i$. To prove this, we first observe that Lemma 3 and the proof of Theorem 7.5 in $[127$ imply that, for all i,

$$
\vec{V}\left(f_{0}, i\right)=\bar{V}\left(f_{0}, 0\right)=\lim _{n \rightarrow \infty} E_{0}, f_{0}\left(\sum_{j=1}^{n} Z_{j}\right) / E_{0, f_{0}}\left(\sum_{j=1}^{n} \tau_{j}\right),
$$

where $z_{k}$ denotes the cost incurred at the $(k-1)$ th review and $T_{k}$ denotes the time be- 
tween the $(k-1)$ th and the $k$ th review. Let $x_{k}$ be the state at the $k$ th review. Since $f_{0}(i)=M$ for al1 $i$ sufficiently large and $\lambda E S_{M}<1$, it follows from Lemma 4 and (2) that

$$
\lim _{k \rightarrow \infty} k^{-1} E_{0, f_{0}}\left(h\left(x_{k}\right)\right)=0
$$

Now the proof of Theorem 7.6 in $[12\rceil$ implies that the right side of (8) equals $g$ (cf. p.727 in [8]). This completes the proof.

\section{REFERENCES}

[1] BEJA, A. \& TELLER, A., "Relevant Policies for Markovian Queueing Systems with Many Types of Service", Management Sci., Vo1. 21 (1975), 1049-1051.

[2] COHEN, J.W., The Single Server Queve, North-Holland, Amsterdam, 1969.

[3] CRABILL, T.B., "Optimal Control of a Service Facility with Variable Exnonential Service Time and Constant Arrival Rate", Management Sci., Vol. 18 (1972), $560-566$.

[4] - "Optimal Hysteric Control of a Stochastic Service System with Variable Service Times and Fixed Switch-Over Costs", University of North Carolina (1973).

[5] DOSHI, B.T., "Continuous-Time Control of Markov Processes on an Arbitrary State Space", Technical Summary Report No. 1468, Mathematical Research Center, University of Wisconsin, Madison, Wisconsin (1974).

[6] HARRISON, J.M., "Countable State Discounted Markovian Decision Processes with Unbounded Rewards", Technical Report No. 17, Department of Operations Research, Stanford University, Stanford, California (1970).

[7] "Discrete Dynamic Programming", Ann. Math. Statist., Vo1. 43 (1972), 636-644.

[8] LIPPMAN, S.A., "Semi-Markov Decision Processes with Unbounded Rewards", Management Sci., Vol. 19 (1973), 717-731.

[9] - "Applying a New Device in the Optimization of Exponential Queueing Systems" (to appear in Operations Res.).

[10] - "On Dynamic Programming with Unbounded Rewards", Management Sci. Vo1. 21 (1975), 1225-1233.

[11] MITCHELL, B., "Optimal Service-Rate Selection in an M/G/1 Queue", Siam J. App Z. Math., Vo1. 24 (1973), 19-35.

[12] ROSS, S.M., Applied Probability Models with Optimization Applications, HoldenDay, Inc., San Francisco, 1970.

[13] SCHASSBERGER, R., "A Note on Optimal Service Selection in a Single Server Queue", Management Sci., Vo1. 21 (1975), 1326-1331.

[14] SOBEL, M.J., "Optimal Operation on Queues", in: Mathematical Methods in Queueing Theory, Lecture Notes in Economics and Mathematical Systems, No. 98, Springer-Verlag, Berlin, 1973.

[15] THATCHER, R.M., "Optimal Single-Channel Service Policies for Stochastic Arrivals", Report ORC 68-16, Operations Research Center, University of California, Berkeley (1968).

[16] TIJMS, H.C., "On a Switch-Over Policy for Controlling the Workload in a Queueing System with Two Constant Service Rates and Fixed Switch-Over Costs", Report BW 45/75, Mathematisch Centrum, Amsterdam (to appear in Zeitschrift für Operations Res.). 
[17] VEINOTT, A.F., Jr., "Optimal Policy in a Dynamic, Single Product, Non-Stationary Inventory Modei with Several Demand Classes", Operations Res., Vo1. 13 (1965), 761-778.

[18] WIDDER, D.V., The LapZace Transform, Princeton University Press, 1946. 